\title{
Amygdala activation and symptom dimensions in obsessive-compulsive disorder
}

Esther Via, Narcís Cardoner, Jesús Pujol, Pino Alonso, Marina López-Solà, Eva Real, Oren Contreras-Rodríguez, Joan Deus, Cinto Segalàs, José M. Menchón, Carles Soriano-Mas and Ben J. Harrison

\section{Background}

Despite knowledge of amygdala involvement in fear and anxiety, its contribution to the pathophysiology of obsessivecompulsive disorder (OCD) remains controversial. In the context of neuroimaging studies, it seems likely that the heterogeneity of the disorder might have contributed to a lack of consistent findings.

\section{Aims}

To assess the influence of OCD symptom dimensions on amygdala responses to a well-validated emotional facematching paradigm.

\section{Method}

Cross-sectional functional magnetic resonance imaging (fMRI) study of 67 patients with OCD and 67 age--, gender- and education-level matched healthy controls.

\section{Results}

The severity of aggression/checking and sexual/religious symptom dimensions were significantly associated with heightened amygdala activation in those with OCD when responding to fearful faces, whereas no such correlations were seen for other symptom dimensions.

\section{Conclusions}

Amygdala functional alterations in OCD appear to be specifically modulated by symptom dimensions whose origins may be more closely linked to putative amygdala-centric processes, such as abnormal fear processing.

\section{Declaration of interest}

None.
The contribution of the amygdala to the pathophysiology of obsessive-compulsive disorder (OCD) remains largely unknown. Although theoretical models continue to suggest a role for this brain region in mediating fear and anxiety processes in individuals with $\mathrm{OCD},{ }^{1,2}$ the available empirical evidence is not compelling. ${ }^{3}$ For example, functional neuroimaging studies of amygdala responsiveness to general threat-related (i.e. fearful) emotional face expressions - a validated experimental context through which the amygdala is reliably activated in humans - have reported increased, decreased or no changes in amygdala responses in patients with OCD $v$. healthy individuals. ${ }^{4-7}$ By comparison, such face-processing tasks have been found to more consistently elicit amygdala hyperactivation in post-traumatic stress disorder, social phobia and generalised anxiety disorder. ${ }^{3}$ Several explanations for these discrepant findings may exist. On the one hand, existing studies of emotional face processing in OCD have been relatively few in number and methodologically diverse. ${ }^{4-7}$ On the other hand, it may be argued that threat-related amygdala responsiveness is simply not of primary relevance to the pathophysiology of OCD as in other related disorders, ${ }^{8}$ or that it is only relevant to certain aspects of its clinical phenomenology. ${ }^{2}$

In the current study, we were particularly interested in addressing this latter possibility; namely, that amygdala function may be more closely related to one or more of the major symptom dimensions of OCD in line with a 'multidimensional' perspective - the idea that OCD can be reliably summarised with a few consistent and temporally stable symptom dimensions. ${ }^{9-12}$ Some existing findings support the usefulness of this approach in the context of neuroimaging studies of patients with OCD. For instance, in an earlier study, ${ }^{13}$ we reported a specific association between amygdala volume reductions and aggression/checking symptoms in individuals with OCD (see also a more recent study by van den Heuvel et al). ${ }^{14}$ In the current study, we sought to examine the influence of OCD symptom dimensions on amygdala function in OCD in the context of a well-validated functional magnetic resonance imaging (fMRI) task of emotional face processing. Recently, we have demonstrated that this task elicits amygdala hyperactivation in OCD patients; ${ }^{7}$ however, because of the sample size of that study we were unable to examine the influence of symptom dimensions. In view of past findings ${ }^{13,14}$ and because our recent study ${ }^{7}$ included patients with a high prevalence of aggression/checking symptoms (76\%) compared with other dimensions (such as contamination/cleaning, 48\%), we sought to test a hypothesis that amygdala function may be specifically modulated by this dimension in people with OCD. Our second objective was to characterise the influence of each symptom dimension on broader regions engaged during the processing of fearful faces, which included areas that we previously identified as hyperactivated in patients with OCD when consciously attending to emotional faces. ${ }^{7}$

\section{Method}

\section{Participants}

A total of 67 adult out-patients were recruited from the Obsessive-Compulsive Disorders Unit of the University Hospital of Bellvitge, Barcelona, Spain (Table 1). Patients were selected from a slightly larger cohort after having satisfied DSM-IV diagnostic criteria $^{15}$ for OCD (for at least 1 year prior to the study) in the absence of relevant medical, neurological and other major psychiatric illness, as well as imaging data quality control checks (see below). Of these patients, 18 (27\%) were included in our original report, ${ }^{7}$ which did not assess the influence of symptom dimensions. Diagnosis was confirmed by two senior psychiatrists through separate interviews 1 month apart using the Structured Clinical Interview for DSM-IV Axis I Disorders (SCID-IV). ${ }^{16}$ No patient met the criteria for Tourette syndrome, any psychotic disorder or psychoactive drug misuse/dependence. Comorbid non-psychotic mood and anxiety disorders were not considered 
to be exclusion criteria provided that OCD was the primary diagnosis. All the patients were taking stable doses of medication during at least a 3-month period coinciding with scanning, except two patients who were medication-free for at least 1 month (Table 2).

Our primary clinical measure of interest was the validated Spanish version of the Dimensional Yale-Brown ObsessiveCompulsive Scale (DY-BOCS), ${ }^{17,18}$ which was used in each patient to rate the severity of six major obsessive-compulsive symptom dimensions: (a) contamination obsessions and cleaning compulsions; (b) obsessions about harm as a result of aggression, injury, violence, natural disasters and/or related compulsions; (c) obsessions concerning sexual, moral and/or religious issues and related compulsions; (d) obsessions about symmetry and/or 'just-right' perceptions, and compulsions to count and/or orderarrange; (e) obsessions and compulsions related to hoarding; (f) miscellaneous obsessions and compulsions (Table 1). Due to concerns about the construct validity of the latter 'miscellaneous' dimension, ${ }^{17}$ it was excluded from subsequent analyses. The DY-BOCS total global severity score was used as a measure of overall illness severity. The Yale-Brown Obsessive Compulsive Scale (Y-BOCS) $)^{19}$ was also included to allow comparability with previous studies.

Patients were appropriately statistically matched for age, gender and years of education to 67 control participants (Table 1). Each control underwent the Structured Clinical Interview for DSM-IV Axis I Disorders non-patient version ${ }^{20}$ to exclude any Axis I psychiatric disorders. None had a personal history of neurological or psychiatric illness. Controls had lower levels of comorbid depression and anxiety symptoms. All the participants provided written informed consent to participate in this study after receiving a complete description of its protocol, which was approved by the Institutional Review Board of the University Hospital of Bellvitge.

\section{Emotional face-matching task}

Participants were assessed using a modified version of the emotional face-matching task originally reported by Hariri et al. ${ }^{21}$ During each $5 \mathrm{~s}$ trial, participants were presented with a target face (centre top) and two probe faces (bottom left and right) and were instructed to match the probe expressing the same emotion to the target by pressing a button in either their left or right hand. A block consisted of six consecutive trials in which the target face was either happy or fearful, and the probe faces included two out of three possible emotional faces (happy, fearful and angry). As a sensorimotor control condition, participants were presented with $5 \mathrm{~s}$ trials of ovals or circles in an analogous configuration and were instructed to match the shape of the probe to the target. Shape stimuli were preferred to neutral faces for the task as the latter may be experienced as emotionally ambiguous or affectively laden, which has been shown to evoke significant activation of amygdala and prefrontal regions. ${ }^{22}$ A total of $6 \times 30 \mathrm{~s}$ blocks of faces ( 3 fearful) and $6 \times 30 \mathrm{~s}$ blocks of the control condition (shapes) were presented interleaved in a pseudorandomised order. A fixation cross was interspersed between each block (online Fig. DS1).

For each trial, response accuracy and response latency (reaction time) were obtained. The paradigm was presented visually on a laptop computer running Presentation software on

\begin{tabular}{|c|c|c|c|c|c|c|}
\hline & OCD group & Control group & $\begin{array}{c}\text { OCD group } \\
n(\%)\end{array}$ & $\begin{array}{c}\text { Control group } \\
n(\%)\end{array}$ & $F_{1,145}$ & $P$ \\
\hline Age, years: mean (s.d.) range & 33.07 (8.50) 19-58 & $32.78(10.22) 19-61$ & $67(100)$ & $67(100)$ & & \\
\hline Gender, male/female: $n$ & $38 / 29$ & $38 / 29$ & $67(100)$ & $67(100)$ & & \\
\hline Education, years: mean (s.d.) range & 12.99 (2.94) 5-19 & $13.34(3.51) 8-22$ & $67(100)$ & $67(100)$ & & \\
\hline Hamilton Rating Scale for Depression (HRSD), mean (s.d.) range & $9.76(5.28) 0-22$ & $0.75(1.62) 0-10$ & $67(100)$ & $61(91)$ & 176.70 & $<0.001$ \\
\hline Hamilton Rating Scale for Anxiety (HRSA), mean (s.d.) range & $12.43(6.54) 2-30$ & $1.67(2.60) 0-17$ & $67(100)$ & $61(91)$ & 154.68 & $<0.001$ \\
\hline Age at onset of OCD, years: mean (s.d.) range & $22.10(8.12) 5-40$ & & $67(100)$ & & & \\
\hline Duration of illness, years: mean (s.d.) range & $11.42(9.18) 1-45$ & & $67(100)$ & & & \\
\hline $\begin{array}{l}\text { Yale-Brown Obsessive-Compulsive Scale (Y-BOCS), } \\
\text { mean (s.d.) range }\end{array}$ & & & $67(100)$ & & & \\
\hline Total $^{a}$ & $21.78(6.07) 10-36$ & & & & & \\
\hline Obsessions & 10.83 (3.08) 5-18 & & & & & \\
\hline Compulsions & 11.02 (3.37) 2-18 & & & & & \\
\hline
\end{tabular}

Dimensional Yale-Brown Obsessive-Compulsive Scale

(DY-BOCS) mean (s.d.) range

Dimensional severity scores: non-zero scores only ${ }^{\mathrm{b}}$

Contamination

Aggression/checking ${ }^{\mathrm{C}}$

Sexual/religious

symmetry/ordering ${ }^{\mathrm{d}}$

Hoarding

Miscellaneous ${ }^{e}$

Global scores ${ }^{a}$

Total symptom severity

Current level of impairment

Global severity score

10.83 (3.08) 5-18

11.02 (3.37) $2-18$

Two-tailed Pearson's $r$ correlations between global measures of the DY-BOCS (total score, global severity, global impairment) and Y-BOCS (obsessions, compulsions and total) showed high correlations (all $r$ ranging between 0.73 and 0.87 and all $P<0.001$ )

b. Non-zero scorers only included in the table. The DY-BOCS severity scores exclude (on a dimension-wise basis) patients whose symptom severity was zero on the rating scale of 0 to 15 c. Note that, although the validity study of the DY-BOCS adopted the labelling 'aggression' for this symptom dimension, we used the term aggression/checking for consistency with previous literature.

d. Symmetry/ordering was the only dimensional severity score that correlated with HRSD and/or HRSA measures (HRSD: $r=0.34, P=0.01 ;$ HRSA: $r=0.43, P<0.001$ ).

e. The miscellaneous dimension was not included in brain-behavioural analyses. 


\begin{tabular}{|c|c|}
\hline & $n(\%)$ \\
\hline \multicolumn{2}{|l|}{ Comorbid mood/anxiety disorder } \\
\hline Major depressive disorder & $4(6)$ \\
\hline Dysthymic disorder & $2(3)$ \\
\hline Bipolar disorder & $0(0)$ \\
\hline Generalised anxiety disorder & $3(5)$ \\
\hline Panic disorder & $4(6)$ \\
\hline Social phobia & $3(5)$ \\
\hline \multicolumn{2}{|l|}{ Medication at time of study } \\
\hline Medication free ( $>4$ weeks) & $2(3)$ \\
\hline Citalopram & $2(3)$ \\
\hline Clomipramine & $29(43)$ \\
\hline Clomipramine with SSRI & $9(13)$ \\
\hline Escitalopram & $7(11)$ \\
\hline Fluoxetine & $7(11)$ \\
\hline Fluoxetine with SSRI & $1(2)$ \\
\hline Fluvoxamine & $4(6)$ \\
\hline Fluvoxamine with SSRI & $3(5)$ \\
\hline Phenelzine & $1(2)$ \\
\hline Sertraline & $1(2)$ \\
\hline Sertraline with SSRI & $1(2)$ \\
\hline With adjunct antipsychotic ${ }^{a}$ & $12(18)$ \\
\hline \multicolumn{2}{|c|}{$\begin{array}{l}\text { SSRI, selective serotonin reuptake inhibitor. } \\
\text { a. Aripiprazole }(n=3) \text {, olanzapine }(n=2) \text {, quetiapine }(n=2) \text {, risperidone }(n=4) \text {, } \\
\text { ziprasidone }(n=1) \text {. }\end{array}$} \\
\hline
\end{tabular}

Windows (Neurobehavioral Systems Inc, Albany, CA, USA, www.neurobehavioralsystems.com). Magnetic resonance imagingcompatible high-resolution goggles were used to display the stimuli. Participants' task responses were registered using a right- and a left-hand response device based on optical fibre transmission. Task performance data were unavailable for one person in the OCD group because of a technical error.

Analyses of task performance data were conducted using a mixed ANOVA, with 'task condition' (fearful faces, happy faces or control condition) as the within-participant variable and 'study group' (controls, individuals with OCD) as the between-participant variable. Response accuracy and reaction times (for correct trials) were estimated and compared separately for each condition. Accuracy was represented as the percentage of correct responses within each condition (out of the non-omission responses). Additionally, we conducted Pearson's correlation analyses (two-tailed) between task performance measures in the OCD group and their DY-BOCS severity ratings. All analyses were performed in Statistical Package for the Social Sciences (SPSS) version 20 on Windows.

\section{Image acquisition and preprocessing}

A 1.5-T Signa Excite system equipped with eight-channel phasedarray head coil and single-shot echoplanar imaging software was used. The functional sequence consisted of gradient recalled acquisition in the steady state (repetition time $(\mathrm{TR})=2000 \mathrm{~ms}$, echo time $(\mathrm{TE})=50 \mathrm{~ms}$ and pulse angle, $90^{\circ}$ ) in a $24 \mathrm{~cm}$ field of view, with a $64 \times 64$ pixel matrix and a slice thickness of $4 \mathrm{~mm}$ (inter-slice gap, $1 \mathrm{~mm}$ ). A total of 22 interleaved sections, parallel to the anterior-posterior commissure line, were acquired to generate 270 whole brain volumes $(9 \mathrm{~min})$, excluding four initial dummy volumes.

Imaging data were pre-processed on a Linux platform running MATLAB version 7 and statistical parametric mapping software (SPM8). Motion correction was performed by aligning (within participant) each time series to the first image volume using a least-squares minimisation and a six-parameter (rigid body) spatial transformation. Translation and rotation estimates $(x, y$, $z$ ) were required to be less than $2 \mathrm{~mm}$ or $2^{\circ}$, respectively, for all participants. Data were normalised to the standard echoplanar imaging (EPI) template provided in SPM software and resliced to $2 \mathrm{~mm}$ isotropic resolution in Montreal Neurological Institute (MNI) space. Functional images were smoothed using a Gaussian filter (full-width at half-maximum $(\mathrm{FWHM})=8 \mathrm{~mm}$ ). All image sequences were routinely inspected for potential normalisation artifacts.

\section{Participant-wise fMRI analysis}

For each participant, a primary task regressor was created by convolving the onset of each of the task condition blocks with a canonical haemodynamic response function and its temporal derivative. Rest-fixation periods served as an implicit task baseline. Maximum likelihood parameter estimates were calculated at each voxel using the general linear model and a first-order autoregressive (AR (1)) model of serial autocorrelations. A high-pass filter was used to remove low-frequency noise $(1 / 128 \mathrm{~Hz})$. First-level contrast images for each participant were carried forward to the group level using the summary statistics approach to random-effects analyses. For this study, the primary task contrast of interest corresponded to the 'matching fearful faces minus control task' condition, given that this contrast provided the most compelling results in our former study. ${ }^{7}$ The condition of 'matching happy faces minus control task' was assessed secondarily in relation to our analyses of symptom dimensions (see below).

\section{Initial between-group fMRI analyses}

A two-sample $t$-test design was used to estimate significant withinand between-group activation results in relation to the primary contrast of interest. For within-group results, a $P_{F D R}<0.05$ with a minimum extend threshold of 10 voxels was used. Betweengroup analyses were performed in two stages. First, an amygdala region-of-interest analysis was performed to test for betweengroups differences in the activation to the task contrast. An amygdala mask was created by applying the cytoarchitectonical probabilistic maps of the amygdala (centromedial, basolateral and superficial subnuclei) included in Anatomy v.1.5 Toolbox in $\mathrm{SPM}^{23}$ to the combined analysis (global conjunction null test as described in SPM $)^{24}$ of control and OCD within-group responses. The defined mask broadly encompassed the bilateral extended amygdala region (824 voxels). The spatial extent threshold for between-group comparisons was determined by 1000 Monte Carlo simulations using AlphaSim ${ }^{25}$ as implemented in the SPM REST toolbox. ${ }^{26}$ The input parameters included an individual voxel threshold probability of 0.01 and a cluster connection radius of $5 \mathrm{~mm}$, at $8 \mathrm{~mm}$ FWHM smoothness. A minimum cluster extent $\left(K_{\mathrm{E}}\right)$ of 12 voxels was estimated to satisfy a $P_{F W E}<0.05$ small-volume corrections.

As a second step, we examined between-group differences across the whole brain volume. In this case, minimum spatial extent thresholds were estimated by applying AlphaSim to extended mask volumes that represented the combined analysis (global conjunction null) of significant within-group effects (size: 79849 voxels, $K_{E}=161$ voxels). The input parameters to AlphaSim were the same as those introduced for the amygdala mask.

\section{fMRI regression analyses of DY-BOCS severity scores}

A multiple regression analysis was used to assess potential associations between the severity of each symptom dimension as a primary regressor of interest and amygdala activation in the OCD group. Associations with symptom dimensions were also assessed across the whole brain. We adopted the same amygdala 
small-volume and whole brain thresholding approach as described above. The mask used for the whole brain analysis corresponded to patients' within-group activation map (size: 82336 voxels, $\mathrm{K}_{E}=66$ voxels).

The influence of each symptom dimension was analysed separately given their mostly orthogonal nature ${ }^{17}$ (online Table DS1), which allowed us to address the possibility that some overlap might occur between dimensions with regard to their influence on amygdala or whole brain activation. Importantly, for each dimension, two covariates were specified: (a) patients' dimension severity score; and (b) a binary (dummy-coded) variable that classified patients into those scoring a zero or a non-zero value (Table 1). This second covariate was included to adjust for a potential categorical weighting to the data that might occur when including a proportion of individuals scoring zero (i.e. 'no symptoms') on a putatively continuous psychometric scale (see also Harrison $e t a l^{27}$ ). Importantly, when adjusting for zero values within each dimension, remaining severity scores became normally distributed (online Fig. DS2). Finally, we also examined potential associations between DY-BOCS total global severity scores and Y-BOCS total scores and patients' brain activation.

\section{Results}

\section{Task performance}

Both groups were accurate overall (mean percentage accuracy: control group: 98.11 (s.d.=2.59); OCD group: 97.11 (s.d.=3.33)) and there was no main effect of group $(F(1,131)=3.75$, $P=0.06)$. However, there was a significant main effect of task condition $(F(1,172)=28.12, P<0.001)$; overall, participants were less accurate when matching fear faces compared with matching shapes and happy faces (all $P<0.001$ ). There was no significant interaction between task condition and study group $(F(1,172)=0.27, P=0.67)$.

Regarding reaction times, there was a significant main effect of group $(F(1,131)=16.30, P<0.001)$, with the OCD group being slower overall than the control group. There was also a significant main effect of condition $(F(1,174)=6.35, P=0.007)$ : reaction times were longer for both face-matching conditions compared with matching shapes $(P<0.001)$ and also when matching fearful compared with happy faces $(P<0.001)$. The OCD group were slower than the control group across all three conditions $(P<0.05)$, but also demonstrated a longer reaction time for the fearful face matching condition, as represented by a significant group $\times$ condition interaction $(F(1,174)=6.35, P=0.007)$.

Within the OCD group, there were no significant linear correlations observed between DY-BOCS dimensions' severity scores (excluding patients with zero scores) or the DY-BOCS global severity scores and task performance measures.

\section{Initial between-group fMRI analyses}

Both groups exhibited significant bilateral activation of the amygdala region when matching fearful emotional face expressions in comparison with matching shapes. Additionally, the groups demonstrated significant activation of other areas commonly engaged by this task, including primary and secondary visual cortices extending to the fusiform gyrus, the hippocampus, premotor (extending to anterior cingulate), lateral prefrontal and orbitofrontal cortices (online Table DS2 and online Fig. DS3).

Between-group analyses indicated significantly greater bilateral amygdala activation in the OCD group (Fig. 1). Significant between-group differences were also characterised in other brain regions. All such differences corresponded to heightened activations in the OCD group compared with the control group. Specifically, patients showed greater activation in secondary visual cortex extended to intraparietal sulcus, right anterior insula cortex, premotor cortex, right orbitofrontal cortex and right middle temporal gyrus. The left amygdala group difference survived whole brain level correction (Fig. 1 and Table DS2).

\section{fMRI regression analyses of DY-BOCS severity scores}

Selective and significant positive correlations between the severity of two symptom dimensions and amygdala activation were observed in the OCD group during the 'matching fearful faces minus shapes' contrast. Of note, these correlations were not observed in our secondary analysis of the 'matching happy faces minus shapes' contrast.

Specifically, the severity of aggression/checking and sexual/ religious symptoms predicted greater activation of the right amygdala when matching fearful face expressions. According to anatomical localisations based on Mai et al, ${ }^{28}$ the main peak of association with aggression/checking symptoms was approximately located in the basolateral amygdala subregion extending to the hippocampus subiculum $\left(x=18, y=-10, z=-16, K_{E}=16\right.$, $Z=3.65)$, whereas the centromedial subregion was mapped to the severity of sexual/religious symptoms $(x=26 \quad y=-10$ $z=-10, K_{E}=28, Z=3.26$ ) (Fig. 2). To explore the influence of anxiety and depression, the scores of the Hamilton Rating Scales for Depression ${ }^{29}$ and Anxiety ${ }^{30}$ were introduced as covariates into these models. In addition, we sought to control for the influence of greater task difficulty with regard to the fearful face-matching condition by including the participants' mean reaction time difference between this condition and the shape-matching condition in further confirmatory analyses. Of note, the observed associations between amygdala activation and OCD symptom dimensions remained significant when controlling for comorbid symptoms of anxiety and depression (aggression/checking $\left(x=18, \quad y=-10, \quad z=-16, K_{E}=12, Z=3.50\right)$, sexual/religious symptoms $\left.\left(x=26, y=-10, z=-10, K_{E}=12, Z=2.90\right)\right)$ and also when controlling for the relatively greater difficulty associated with matching fearful faces compared with the control trials (aggression/checking $\left(x=18, y=-10, z=-16, K_{E}=12, Z=3.55\right)$, sexual/religious $\left.\left(x=26, y=-10, \quad z=-10, K_{E}=31, \quad Z=3.36\right)\right)$ (online Fig. DS4).

Other brain regions also emerged as significantly positively and negatively correlated with the severity of these two dimensions, but not other dimensions, in relation to matching fearful faces. Greater severity of aggression/checking symptoms was positively correlated with activation of the dorsal anterior cingulate cortex and negatively correlated with the posteriorinferior middle temporal gyrus. Greater severity of sexual/religious symptoms was additionally positively correlated with activation of the left premotor cortex and negatively correlated with activation of the primary and secondary visual cortex and the right fusiform gyrus (Fig. 2).

Finally, DY-BOCS total global severity was negatively correlated with the activation of visual cortex regions, extending to the intraparietal cortex. These correlations also emerged when using the total Y-BOCS score, although the regional clusters were less extensive (online Fig. DS5).

\section{Discussion}

Although the amygdala remains theoretically important to pathophysiological models of OCD, empirical studies such as those based on neuroimaging have not provided consistent support for its involvement in the disorder. This study sought to test the idea that putative amygdala dysfunction may be more 

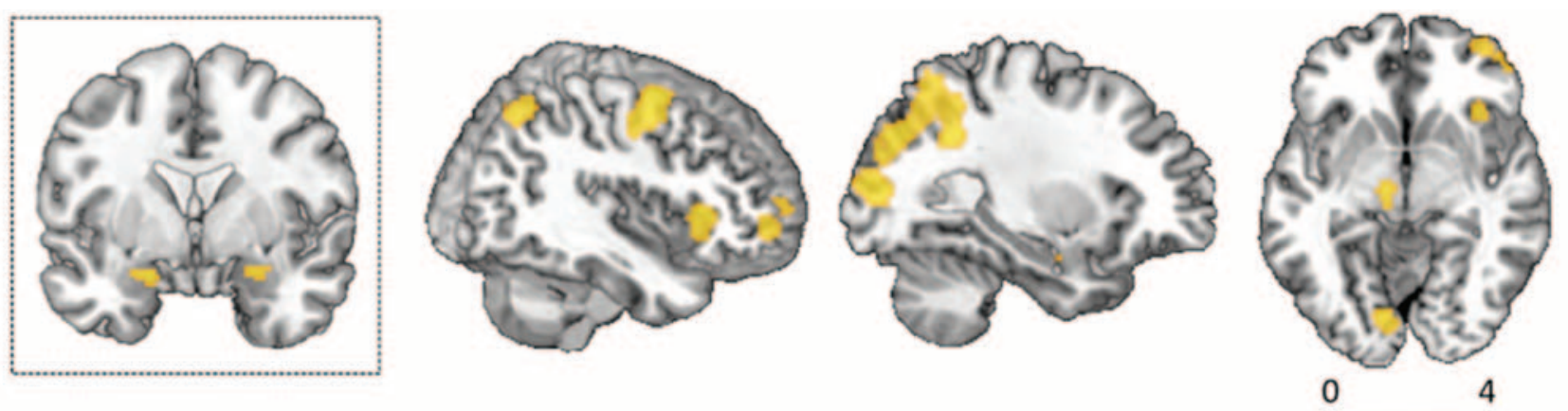

Fig. 1 Amygdala and whole brain between-group differences (obsessive-compulsive disorder (OCD) group > healthy control group) during the performance of the task (fearful faces minus control task).

Results for amygdala-small-volume correction (SVC) approach (images within the dashed-line box) and whole brain approach are represented at $P_{F W E}<0.05$ AlphaSim corrected. Images Results for amygdala-small-Volume correction (SVC) approach (Images within the dashed-line box) and whole brain approach are represented at $P_{F W E}<0.05$ AlphaSim corrected. Images
are represented in standard neuroanatomical space (Montreal Neurological Institute (MNI)) and in neurological convention (i.e. right hemisphere is displayed on the right), colour bar are represented in standard neuroanatomical space (Montreal Neurological Institute (MNI)) and in neurological convention (i.e. right hemisphere is displayed on the right), colour
represents T-value. Peak coordinates and statistics corresponding to the amygdala-SVC - right amygdala: $X=24, y=2, Z=-16, K_{E}=12, Z=2.88 ;$ left amygdala: $x=-18, y=-2$, $Z=-16, K_{E}=103, Z=3.51$. Whole brain peak coordinates are included in online Table DS2.
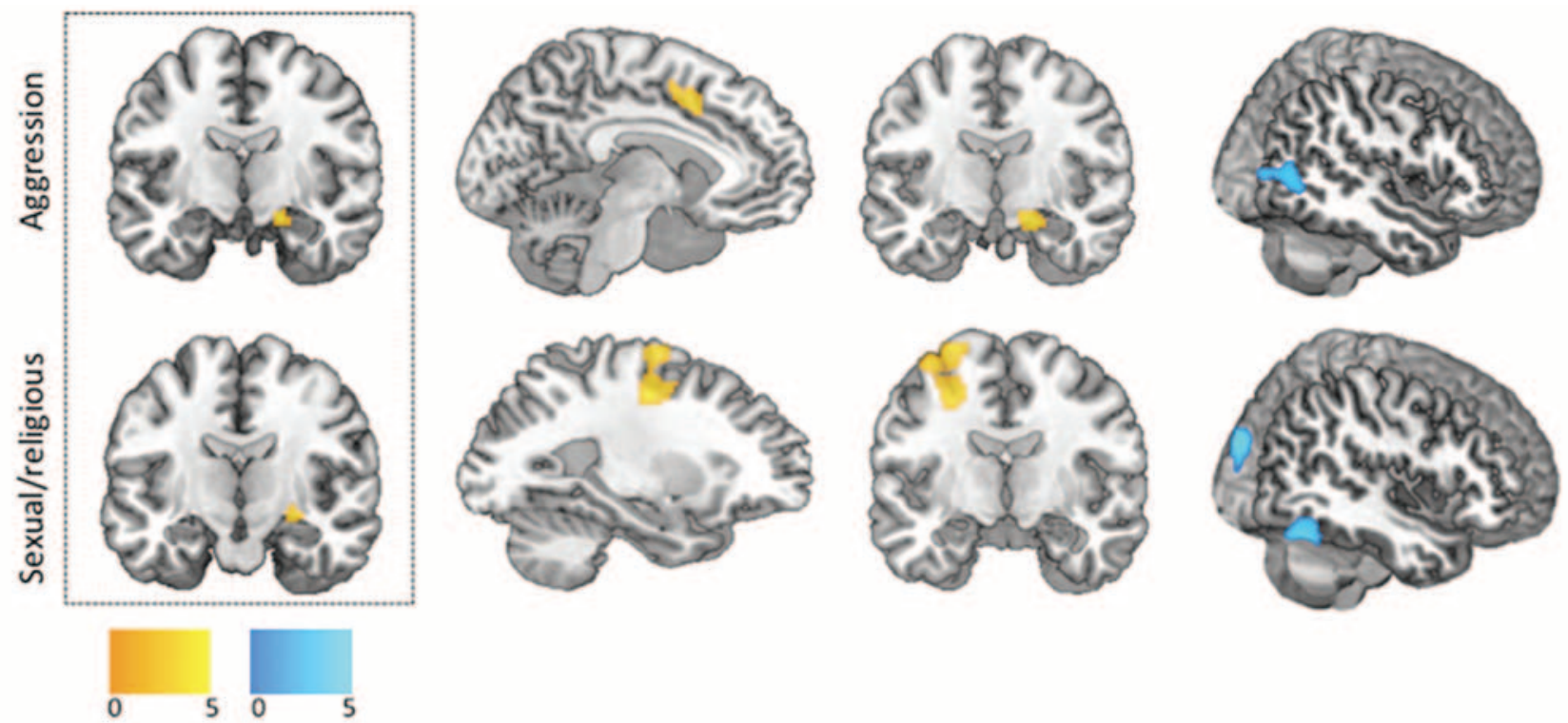

Fig. 2 Amygdala and whole brain correlations between the Dimensional Yale-Brown Obsessive-Compulsive Scale (DY-BOcS) scores and brain activations during the performance of the task (fearful faces minus control task).

Images are represented in standard neuroanatomical space (Montreal Neurological Institute (MNI)) and in neurological convention (i.e. right hemisphere is displayed on the right). Colour bar represents the T-value. Positive correlations in yellow, negative correlations in blue. Results for the correlations using the amygdala-small-volume correction (SVC) approach (images within the dashed-line box) and whole brain are represented at a $P_{F W E}<0.05$ AlphaSim corrected. Peak coordinates and statistics (KE $=$ cluster size, Z): amygdala-SVC approach - aggression/checking: $x=18, y=-10, z=-16, K_{E}=16, Z=3.65$; sexual/religious: $x=26, y=-10 z=-10, K_{E}=28, Z=3.26$. Whole brain approach - aggression/checking (from left to right): anterior cingulate cortex: $x=-6, y=12, Z=44, K_{E}=170, Z=3.16$; amygdala: $x=20, y=-14, Z=-16, K_{E}=185, Z=4.00$; middle temporal gyrus: $x=46, y=-76$, $Z=8, K_{E}=240, Z=2.54$; sexual/religious (from left to right): left premotor cortex, sagittal view $-26, y=-10, Z=46, K_{E}=422, Z=3.44$; left premotor cortex, coronal view; extended visual areas: middle occipital cortex $x=26, y=-90, z=26, K_{E}=293, Z=3.69$, lingual gyrus: $x=-2, y=88, z=-2, K_{E}=315, Z=3.63$ and fusiform gyrus: $x=44, y=-60, z=-22$, $K_{E}=217, Z=3.28$

closely related to one or more of the major symptom dimensions of OCD in an attempt to reconcile these past findings. In general, this idea is consistent with one of the key predictions of the 'multidimensional model', which has proposed that OCD symptom dimensions may differentially reflect the disturbance of brain 'threat detection' systems involving frontal, striatal and limbic regions. ${ }^{31,32}$ The current study lends some support to this idea by demonstrating a selective modulation of amygdala function in participants with OCD in relation to aggression/checking and sexual/religious symptoms but not other symptom dimensions. Whereas the association with aggression/checking symptoms was predicted on the basis of our prior work ${ }^{7}$ and previous structural imaging studies, ${ }^{13,14}$ the association with sexual/religious symptoms may also be considered intuitive, considering that both of these dimensions have been grouped together under the umbrella of unacceptable thoughts, factor I or pure obsessions. ${ }^{9,12}$

Associations between the severity of aggressive and sexual/ religious symptoms and amygdala activation were characterised in the context of a fearful emotional face-matching task. Although the task itself is not considered to be fear eliciting, the processing of fearful faces is known to broadly engage components of the classical 'fear circuitry' ${ }^{21,33}$ including the amygdala. ${ }^{34}$ Thus, it may be hypothesised that disturbances within this circuitry are more relevant to the development and maintenance of these particular symptom dimensions in people with OCD. These results also raise the question of whether these two dimensions might have greater biological overlap with other anxiety disorders, given the stronger existing link between these disorders and 
amygdala fear circuit dysfunction. ${ }^{3}$ This idea fits with previous suggestions of a phenomenological relationship between some symptom dimensions and other anxiety disorders. ${ }^{2}$ Of note, the presence of comorbid anxiety disorders and anxiety symptoms did not account for the observed relationships between the two symptom dimensions and amygdala activation in our patients.

It is notable that the severity of aggression/checking and sexual/religious symptoms modulated somewhat distinct areas of the extended amygdala complex and differentially predicted activation in other task-relevant regions. Specifically, the severity of aggression/checking symptoms predicted the activation of the amygdala and the dorsal anterior cingulate cortex. Together with the amygdala, the dorsal anterior cingulate is also implicated in the processing (evaluation) of fear-related stimuli. ${ }^{35}$ Both structures are also implicated in other relevant functions, such as interoceptive awareness and emotional arousal (together with the insula) - forming a network that is thought to be dysregulated in anxiety-prone individuals ${ }^{36}$ and whose activity may underlie the emergence of anxious affect, worry and avoidance behaviour. ${ }^{36}$ Other evidence points to the relevance of amygdala and anterior cingulate in the evaluation of remote fears and threat estimation. ${ }^{35,37}$ Interestingly, threat estimation beliefs have also been shown to predict aggression/checking symptoms in people with OCD, ${ }^{38}$ and, in general, appear to be one domain of obsessional beliefs that is more common between patients with OCD and patients with other anxiety disorders. ${ }^{39}$ It is also interesting to note that although the anterior cingulate is reported as being generally hyperactive in people with $\mathrm{OCD},{ }^{40-43}$ certain studies have associated these findings to the severity or provocation of aggression/checking symptoms and anxiety. ${ }^{31,41,44}$

Sexual/religious symptoms were also correlated with activation of the amygdala and with the left premotor cortex. The precise anatomical location of this amygdala cluster was slightly different to that observed with aggression/checking symptoms; the former being associated more with the centromedial amygdala, and the latter being associated more with the basal amygdala. These findings may hint at a certain level of amygdala functional specialisation with regard to these symptom dimensions, although this suggestion is made cautiously, taking into account the spatial resolution of our data.

Although hyperactivation of premotor cortex has been previously related to obsessional slowness, ${ }^{45}$ some evidence suggests its relevance in the response to threat, as a preparatory motor response of the individual perceiving danger. ${ }^{46}$ In addition, its activity may be particularly relevant in the context of functional alterations of the amygdala. ${ }^{47}$ Its specific relationship to sexual/religious symptoms, however, remains to be clarified.

Negative associations were also found between the severity of these dimensions and areas related to visual perception, namely the middle temporal gyrus (its posterior-inferior portion) in the case of aggression/checking symptoms and extended visual cortex areas in the case of sexual/religious symptoms. It is interesting that the DY-BOCS and Y-BOCS overall severity scores also emerged as negatively correlated with primary visual and intraparietal cortex regions. Taken together, these findings suggest a common converging influence of multiple OCD severity measures on the engagement of task-relevant visual processing areas. Precisely how the modulation of 'lower order' visual regions might impact on amygdala function in people with OCD is unclear at this point, but likely involves complex interactions between the so-called dorsal and ventral visual attention pathways. The task-specific involvement of dorsal attention regions and their heightened engagement in patients with OCD is discussed at greater length in Cardoner et al. ${ }^{7}$

\section{Strengths and limitations}

The major strengths of this study relate to its large sample, the use of a task that has proven reliable for activating the amygdala in healthy individuals and clinical populations and the use of a psychometric assessment specifically designed to assess OCD symptom dimensions. ${ }^{17}$ However, a number of possible limitations of this work should be considered. First, most of the patients were medicated with a selective serotonin reuptake inhibitor or clomipramine-based treatment strategy. Although on the basis of existing evidence such treatments may be expected to reduce rather than augment amygdala activation, ${ }^{48}$ their potential influence on our findings cannot be ruled out, particularly with regard to symptom dimension associations. Second, our results are limited by the specificity of the task and therefore could be meaningfully advanced by novel experiments designed, for example to assess fear-conditioning processes in patients with OCD. It is possible that in the context of such tasks, associations between putative 'fear circuit' regions and other hypothesised 'limbic-mediated' symptom dimensions, such as contamination/cleaning symptoms, ${ }^{2}$ may be revealed. Finally, although our results may suggest some biological overlap between aggression/checking and sexual/religious symptoms and other anxiety disorders, direct comparisons will be necessary and useful for clarifying these notions.

\section{Implications}

Our results suggest that amygdala functional alterations are present in patients with OCD, but appear to be relevantly modulated by certain symptom dimensions. These findings may have implications for the conceptualisation and treatment of OCD. First, our results lend further support to the idea that OCD symptom dimensions are neurobiologically valid clinical phenotypes that deserve attention in imaging, genetic and other studies of OCD.,17 Second, although the idea that certain dimensions might be biologically closer to other anxiety disorders appears to contradict recent proposals regarding the diagnostic classification of $\mathrm{OCD},{ }^{49}$ it raises important questions that will only be resolved in future research. In particular, it has raised the question of whether processes such as abnormal fear learning and extinction, which are implicated in the pathogenesis of common anxiety disorders, are equally as relevant to the development and maintenance of certain OCD symptom dimensions. It would be particularly interesting to explore these questions in the context of treatment-oriented research, especially through studies focused on the provision of cognitive-behavioural therapies for OCD, which putatively target fear learning and extinction processes.

\section{Funding}

This study was supported in part by the Carlos III Health Institute (PI05/0884, PI07/1029, PI09/01331, PI10/01753, PI10/01003, CP10/00604 and CIBER-CB06/03/0034) and by the Agency of University and Research Funding Management of the Catalan Government (AGAUR; 2009SGR1554 and 2009SGR1450). M.L.-S. is supported by a Beatriu de Pinós Modalitat-A fellowship awarded by AGAUR (fellowship number: 2010 BP_A 00136). ER is supported by a Río Hortega contract from the carlos III Health Institute (ID. CM11/ 00077). C.S.M is funded by a Migul servet contract from the Carlos II Health institute (CP10/00604). B.J.H is supported by a National Health and Medical Research Council of (CP10/00604). B.J.H is supported by a National Health and Medical Research Council of
Australia (NHMRC) Clinical Career Development Award (I.D. 628509).

\section{Acknowledgements}

The authors thank all of the study participants as well as the staff from the Department of Psychiatry, Hospital Universitari de Bellvitge. 
Esther Via, MD, Department of Psychiatry, Bellvitge University Hospital-IDIBELL, Hospitalet de Llobregat, Barcelona, Spain, and Melbourne Neuropsychiatry Centre, Department of Psychiatry \& Melbourne Health, The University of Melbourne, National Neuroscience Facility, Melbourne, Australia; Narcís Cardoner, MD, PhD, Department of Psychiatry, Bellvitge University Hospital-IDIBELL, Hospitalet de Llobregat, Barcelona, Spain, Carlos III Health Institute, CIBERSAM, Spain and Department of Clinical Sciences, School of Medicine, University of Barcelona, Barcelona, Spain; Jesús Pujol, MD, MRI Research Unit, CRC Mar, Hospital de Mar, Barcelona, Spain; Pino Alonso, MD, PhD, Department of Psychiatry, Bellvitge University Hospital-IDIBELL, Hospitalet de Llobregat, Barcelona, Spain, Carlos III Health Institute, CIBERSAM, Spain, and Department of Clinical Sciences, School of Medicine, University of Barcelona, Barcelona, Spain; Marina López-Solà, PhD, MRI Research Unit, CRC Mar, Hospital de Mar, Barcelona, Spain and Department of Psychology and Neuroscience, University of Colorado at Boulder, Boulder, Colorado, USA Eva Real, MD, Department of Psychiatry, Bellvitge University Hospital-IDIBELL, Hospitalet de Llobregat, Barcelona, Spain and Carlos III Health Institute, CIBERSAM, Spain; Oren Contreras-Rodríguez, PhD, Carlos III Health Institute, CIBERSAM, Spain and MRI Research Unit, CRC Mar, Hospital de Mar, Barcelona, Spain; Joan Deus, PhD, MRI Research Unit, CRC Mar, Hospital de Mar, Barcelona, and Department of Clinical and Health Psychology, Autonomous University of Barcelona, Barcelona, Spain; Cinto Segalàs, MD, PhD, Department of Psychiatry, Bellvitge University Hospital-IDIBELL, Hospitalet de Llobregat, Barcelona, Spain, and Carlos II Health Institute, CIBERSAM, Spain; José M. Menchón, MD, PhD, Department of Psychiatry, Bellvitge University Hospital-IDIBELL, Hospitalet de Llobregat, Barcelona, Spain, Carlos III Health Institute, CIBERSAM, Spain, and Department of Clinical Sciences, School of Medicine, University of Barcelona, Barcelona, Spain; Carles Soriano-Mas, PhD, Department of Psychiatry, Bellvitge University Hospital-IDIBELL, Hospitalet de Llobregat, Barcelona, Spain and Carlos III Health Institute, CIBERSAM, Spain; Ben J. Harrison, PhD, Melbourne Neuropsychiatry Centre, Department of Psychiatry \& Melbourne Health, The University of Melbourne, Melbourne, Australia

Correspondence: Ben J. Harrison, PhD, Melbourne Neuropsychiatry Centre, Department of Psychiatry \& Melbourne Health, The University of Melbourne, c/o National Neuroscience Facility, 161 Barry Street, Carlton, 3053, Melbourne, Australia.Email: habj@unimelb.edu.au.

First received 8 Nov 2012, final revision 16 Apr 2013, accepted 8 May 2013

\section{References}

1 Milad MR, Rauch SL. Obsessive-compulsive disorder: beyond segregated cortico-striatal pathways. Trends Cogn Sci 2012; 16: 43-51.

2 Mataix-Cols D, van den Heuvel OA. Common and distinct neural correlates of obsessive-compulsive and related disorders. Psychiatr Clin North Am 2006; 29: 391-410, viii.

3 Shin LM, Liberzon I. The neurocircuitry of fear, stress, and anxiety disorders Neuropsychopharmacology 2010; 35: 169-91.

4 Cannistraro PA, Wright $\mathrm{Cl}$, Wedig MM, Martis B, Shin LM, Wilhelm S, et al. Amygdala responses to human faces in obsessive-compulsive disorder. Bio Psychiatry 2004; 56: 916-20.

5 Lawrence NS, An SK, Mataix-Cols D, Ruths F, Speckens A, Phillips ML. Neura responses to facial expressions of disgust but not fear are modulated by washing symptoms in OCD. Biol Psychiatry 2007; 61: 1072-80.

6 Britton JC, Stewart SE, Killgore WD, Rosso IM, Price LM, Gold AL, et al. Amygdala activation in response to facial expressions in pediatric obsessivecompulsive disorder. Depress Anxiety 2010; 27: 643-51.

7 Cardoner N, Harrison BJ, Pujol J, Soriano-Mas C, Hernandez-Ribas R, Lopez Sola $M$, et al. Enhanced brain responsiveness during active emotional face processing in obsessive compulsive disorder. World J Biol Psychiatry 2011; 12: 349-63.

8 Phillips KA, Stein DJ, Rauch SL, Hollander E, Fallon BA, Barsky A, et al. Should an obsessive-compulsive spectrum grouping of disorders be included in DSM-V? Depress Anxiety 2010; 27: 528-55.

9 Leckman JF, Grice DE, Boardman J, Zhang H, Vitale A, Bondi C, et al. Symptoms of obsessive-compulsive disorder. Am J Psychiatry 1997; 154 911-7.

10 Mataix-Cols D, Rosario-Campos MC, Leckman JF. A multidimensional mode of obsessive-compulsive disorder. Am J Psychiatry 2005; 162: 228-38.

11 Rufer M, Grothusen A, Mass R, Peter H, Hand I. Temporal stability of symptom dimensions in adult patients with obsessive-compulsive disorder. $J$ Affect Disord 2005; 88: 99-102.

12 Baer L. Factor analysis of symptom subtypes of obsessive compulsive disorder and their relation to personality and tic disorders. J Clin Psychiatry 1994; 55 (suppl): 18-23.

13 Pujol J, Soriano-Mas C, Alonso P, Cardoner N, Menchon JM, Deus J, et al. Mapping structural brain alterations in obsessive-compulsive disorder. Arch Gen Psychiatry 2004; 61: 720-30.

14 van den Heuvel $\mathrm{OA}$, Remijnse $\mathrm{PL}$, Mataix-Cols $\mathrm{D}$, Vrenken $\mathrm{H}_{\text {, }}$ Groenewegen $\mathrm{HJ}$, Uylings $\mathrm{HB}$, et al. The major symptom dimensions of obsessive-compulsive disorder are mediated by partially distinct neural systems. Brain 2009; 132: 853-68.

15 American Psychiatric Association. Diagnostic and Statistical Manual of Mental Disorders (4th edn) (DSM-IV). APA, 1994.

16 First MB, Spitzer RL, Gibbon M, Williams JBW. Structured Clinical Interview for DSM-IV Axis I Disorders-Clinician Version (SCID-CV). American Psychiatric Press, 1997.

17 Rosario-Campos MC, Miguel EC, Quatrano S, Chacon P, Ferrao Y, Findley D, et al. The Dimensional Yale-Brown Obsessive-Compulsive Scale (DY-BOCS): an instrument for assessing obsessive-compulsive symptom dimensions. Mol Psychiatry 2006; 11: 495-504.

18 Pertusa A, Jaurrieta N, Real E, Alonso P, Bueno B, Segalas C, et al. Spanish adaptation of the Dimensional Yale-Brown Obsessive-Compulsive Scale. Compr Psychiatry 2010; 51: 641-8.

19 Goodman WK, Price LH, Rasmussen SA, Mazure C, Delgado P, Heninger GR, et al. The Yale-Brown Obsessive Compulsive Scale. II. Validity. Arch Gen Psychiatry 1989; 46: 1012-6.

20 First MB, Spitzer RL, Gibbon M, Williams JB. Structured Clinical Interview for DSM-IV-RS Axis 1 Disorders - Non-patient edition (SCID-I/NP). Biometrics Research, New York State Psychiatric Institute, 2007.

21 Hariri AR, Bookheimer SY, Mazziotta JC. Modulating emotional responses: effects of a neocortical network on the limbic system. Neuroreport 2000; 11: 43-8.

22 Schwartz CE, Wright Cl, Shin LM, Kagan J, Whalen PJ, McMullin KG, et al. Differential amygdalar response to novel versus newly familiar neutral faces: a functional MRI probe developed for studying inhibited temperament. Biol Psychiatry 2003; 53: 854-62.

23 Eickhoff S, Stephan KE, Mohlberg H, Grefkes C, Fink GR, Amunts K, et al. A new SPM toolbox for combining probabilistic cytoarchitectonic maps and functional imaging data. Neurolmage 2005; 25: 1325-35.

24 Friston KJ, Penny WD, Glaser DE. Conjunction revisited. Neuroimage 2005; 25: 661-7.

25 Ward BD. Simultaneous Inference for FMRI Data. NIMH, 2000 (http:// afni.nimh.nih.gov/pub/dist/doc/manual/AlphaSim.pdf)

26 Song XW, Dong ZY, Long XY, Li SF, Zuo XN, Zhu CZ, et al. REST: a toolkit for resting-state functional magnetic resonance imaging data processing. PLOS One 2011; 6: e25031.

27 Harrison BJ, Pujol J, Cardoner N, Deus J, Alonso P, Lopez-Sola M, et al. Brain corticostriatal systems and the major clinical symptom dimensions of obsessive-compulsive disorder. Biol Psychiatry 2013; 73: 321-8.

28 Mai JK, Voss T, Paxinos G. Atlas of the Human Brain. Academic Press, 2007

29 Hamilton M. A rating scale for depression. J Neurol Neurosurg Psychiatry 1960; 23: 56-62.

30 Hamilton M. The assessment of anxiety states by rating. Br J Med Psychol 1959; 32: 10.

31 Mataix-Cols D, Wooderson S, Lawrence N, Brammer MJ, Speckens A, Phillips $\mathrm{ML}$. Distinct neural correlates of washing, checking, and hoarding symptom dimensions in obsessive-compulsive disorder. Arch Gen Psychiatry 2004; 61: 564-76.

32 Leckman JF, Mataix-Cols D, Rosario-Campos MC. Symptom dimensions in OCD: developmental and evolutionary perspectives and Reply to Taylor: combined dimensional and categorical perspectives as an integrative approach to OCD. In Concepts and Controversies in Obsessive-Compulsive Disorder (eds JS Abramowitz, AC Houts): 3-21, 43-47. Springer Science, 2005.

33 Haxby JV, Hoffman EA, Gobbini MI. The distributed human neural system for face perception. Trends Cogn Sci 2000; 4: 223-33.

34 LeDoux J. The emotional brain, fear, and the amygdala. Cell Mol Neurobiol 2003; 23: 727-38.

35 Fiddick $\mathrm{L}$. There is more than the amygdala: potential threat assessment in the cingulate cortex. Neurosci Biobehav Rev 2011; 35: 1007-18.

36 Paulus MP, Stein MB. An insular view of anxiety. Biol Psychiatry 2006; 60: 383-7.

37 Maren S. Neuroscience. The threatened brain. Science 2007; 317: 1043-4.

38 Wheaton MG, Mahaffey B, Timpano KR, Berman NC, Abramowitz JS. The relationship between anxiety sensitivity and obsessive-compulsive symptom dimensions. J Behav Ther Exp Psychiatry 2012; 43: 891-6.

39 Tolin DF, Worhunsky P, Maltby N. Are "obsessive" beliefs specific to OCD?: a comparison across anxiety disorders. Behav Res Ther 2006; 44: 469-80.

40 Baxter Jr LR, Schwartz JM, Mazziotta JC, Phelps ME, Pahl JJ, Guze BH, et al. Cerebral glucose metabolic rates in nondepressed patients with obsessive-compulsive disorder. Am J Psychiatry 1988; 145: 1560-3. 
41 Perani D, Colombo C, Bressi S, Bonfanti A, Grassi F, Scarone S, et al. [18F]FDG PET study in obsessive-compulsive disorder. A clinical/metabolic correlation study after treatment. Br J Psychiatry 1995; 166: 244-50.

42 Machlin SR, Harris GJ, Pearlson GD, Hoehn-Saric R, Jeffery P Camargo EE. Elevated medial-frontal cerebral blood flow in obsessive-compulsive patients: a SPECT study. Am J Psychiatry 1991; 148: 1240-2.

43 Swedo $\mathrm{SE}$, Schapiro MB, Grady $\mathrm{CL}$, Cheslow DL, Leonard $\mathrm{HL}$, Kumar A, et al. Cerebral glucose metabolism in childhood-onset obsessive-compulsive disorder. Arch Gen Psychiatry 1989; 46: 518-23.

44 Phillips ML, Marks IM, Senior C, Lythgoe D, O'Dwyer AM, Meehan O, et al. A differential neural response in obsessive-compulsive disorder patients with washing compared with checking symptoms to disgust. Psychol Med 2000; 30: $1037-50$.
45 Sawle GV, Hymas NF, Lees AJ, Frackowiak RS. Obsessional slowness. Functional studies with positron emission tomography. Brain 1991; 114 2191-202.

46 Phelps EA, O'Connor KJ, Gatenby JC, Gore JC, Grillon C, Davis M. Activation of the left amygdala to a cognitive representation of fear. Nat Neurosci 2001; 4: 437-41.

47 Becker B, Mihov Y, Scheele D, Kendrick KM, Feinstein JS, Matusch A, et al. Fear processing and social networking in the absence of a functional amygdala. Biol Psychiatry 2012; 72: 70-7.

48 Del-Ben CM, Deakin JF, McKie S, Delvai NA, Williams SR, Elliott R, et al. The effect of citalopram pretreatment on neuronal responses to neuropsychological tasks in normal volunteers: an FMRI study. Neuropsychopharmacology 2005; 30: 1724-34.

49 American Psychiatric Association. Diagnostic and Statistical Manual of Mental Disorders (5th edn) (DSM-5). APA, 2013.

\section{reflection On Laing's The Divided Self}

\section{Sandy Robertson}

Re-reading The Divided Self it is hard to recapture the glamour and controversy which surrounded its author in the heyday of antipsychiatry. Fame, drugs and alcohol gave us a different Ronald Laing whose views became increasingly estranged from the clinical mainstream; but his first book is a dense and scholarly work whose complexity derives from his immediate clinical experience, in the analytic setting and in acute wards, combined with an enormous range of reading in existential philosophy and psychoanalysis.

To understand its impact one must recall that the orthodoxy of the age was that psychiatry should be a natural science and therefore ruthlessly objective. Laing reminded us that the subject matter of psychiatry is not only the experience of the patient but that of the psychiatrist; both are embodied subjects, to use Eric Matthews' phrase, and the clinical encounter is a meeting between persons. He criticised the neutral stance of the psychoanalyst and Freudian theory which, like the Medusa's head, turned the terrors of the unconscious to stone. It was this humanising of the clinical situation which won him many adherents among patients and those who identified with them.

Laing's stated aim in the book is to 'make the process of going mad understandable' and his unifying theme is that of ontological insecurity which he believes is central to schizoid and schizophrenic experience. This is a fragile and endangered sense of self which the individual tries to shore up with a variety of defensive stratagems that become increasingly self-defeating until they break down into frank psychosis. Some patients, for instance, only experience themselves as real to the extent that they are experienced by others, but at the same time feel others' experience of them to be intrusive and persecutory. Such complex and paradoxical phenomena are explored with sensitivity and compassion in a series of detailed case histories.

The sceptical psychiatrist, while admiring Laing's analytic and existential insights, may doubt their relevance to those diagnosed with schizophrenia in conventional diagnostic systems. They may also have reservations about the direction of causality: schizophrenia threatens selfhood but this need not mean that ontological insecurity is the cause of schizophrenia. Laing, however, was concerned here with understanding rather than cause; only in later works did he suggest that the cause of schizophrenia lay in family dynamics. Still less is there any suggestion here that the experience of madness is anything other than destructive.

Laing's legacy has had little direct effect on clinical psychiatry. The treatment of psychosis remains largely chemical and social despite the best efforts of cognitive therapists. Its indirect effects, however, have been immense. The anti-psychiatry movement, with which Laing's name is often associated (though he repudiated the label), played a large part in stimulating the thriving disciplines of philosophy and ethics in psychiatry. Patients have found their voice through the 'user' movement and are in dialogue with mental health professionals at all levels. Psychiatrists recognise that content matters, above all to patients, and hopefully are motivated to pursue meaning before concluding that a mental state is truly non-understandable in Jaspers' sense. 